\title{
The Structure of Charge Density Wave Phase Transitions in Atomically Thin Materials
}

Robert Hovden ${ }^{1}$, Suk Hyun Sung ${ }^{1}$, Noah Schnitzer ${ }^{2}$, Steve Novakov ${ }^{1}$, Ismail El Baggari ${ }^{2}$, Benjamin Savitzky $^{3}$, John Heron ${ }^{1}$ and Lena Kourkoutis ${ }^{2}$

${ }^{1}$ University of Michigan, Ann Arbor, Michigan, United States, ${ }^{2}$ Cornell University, Ithaca, New York, United States, ${ }^{3}$ National Center for Electron Microscopy (NCEM), Molecular Foundry, Lawrence Berkeley National Laboratory, Berkeley, California, United States

Charge-density-wave (CDW) transition metal dichalcogenides (TMDs) are materials of historic interest [1] which have seen a resurgence in attention following successful exfoliation to few layer thicknesses [2]. These charge density waves mediate metal-insulator transitions and compete with superconductivity. Previous work has revealed dopants, pressure, temperatures, and electrical biases can manipulate the transition between CDW phases [3]-[5]. Cooling across the transition temperature $\left(\mathrm{T}_{\mathrm{c}}\right)$ in $1 \mathrm{~T}-\mathrm{TaS}_{2}$, causes a reshaping of the CDW and its associated periodic lattice distortions (PLDs) that results in a resistivity change. When reduced to low dimensions $(<10 \mathrm{~nm}), 2 \mathrm{D}$ CDW resistivity changes offer new mechanisms for electronic switching and nonvolatile memory[5]. Although various techniques have been demonstrated to manipulate charge order, a fundamental understanding is still lacking, and the methods used are incompatible with device fabrication. Mounting evidence indicates that nanoscale spatial inhomogeneity between competing phases plays a fundamental role in complex electronic systems quite broadly. In this work we use high-resolution scanning transmission electron microscopy (STEM) to measure lattice distortions concomitant with CDWs and correlate their structure with electronic and temperature control to unveil the microscopic nature of charge ordered phases in thin van der Waals materials.

Here, S/TEM is correlated with electrical and temperature measurements on nanostructured CDW devices to provide a microscopic understanding of the metal-to-insulator transitions that accompany commensuration of charge waves with the underlying lattice. Our work stabilizes $2 \mathrm{D}$ charge density waves down to low-temperature $(93 \mathrm{~K})$ and correlates their electronic properties with atomic structure $[5,6]$. Figure 1 shows the atomic structure of the commensurate CDW lattice in ultrathin $1 \mathrm{~T}-\mathrm{TaS}_{2}$. In a charge ordered bulk oxide, we recently found that a phase change in charge ordered materials is not described by an incommenseration of the CDW with the underlying lattice, but rather associated with local phase-shifts in the CDW wavevector causing degradation of long-range CDW order[7,8]. We report similar behavior in $\mathrm{TaS}_{2}$, however additional disorder - such as polytype transformations, stacking order, and CDW structure - can dramatically impact electronic resistivity both in-plane and out of plane. Figure 2 shows the device structure of a $\mathrm{TaS}_{2}$ flake and two resistance curves taken from a single crystal (pink) and a crystal with disordered stacking and bong coordination (blue)_illustrating noticeably different resistance and phase transitions [9]. 



Figure 1. PLD ordering and atomic resolution STEM imaging of thin commensurate phase 1T- TaS2. (left) Within a layer, the commensurate PLD / CDW contains 13 Ta sites in a hexagonal supercell (sulfur not shown). (right) At 95K the commensurate structure is visible in HAADF images of thicker exfoliated flakes suggesting at least partial ordering of the CDW in the out-of-plane direction. The superlattice is highlighted by a blue parallelogram with $1.2 \mathrm{~nm}$ sides. Figure adapted from [6].
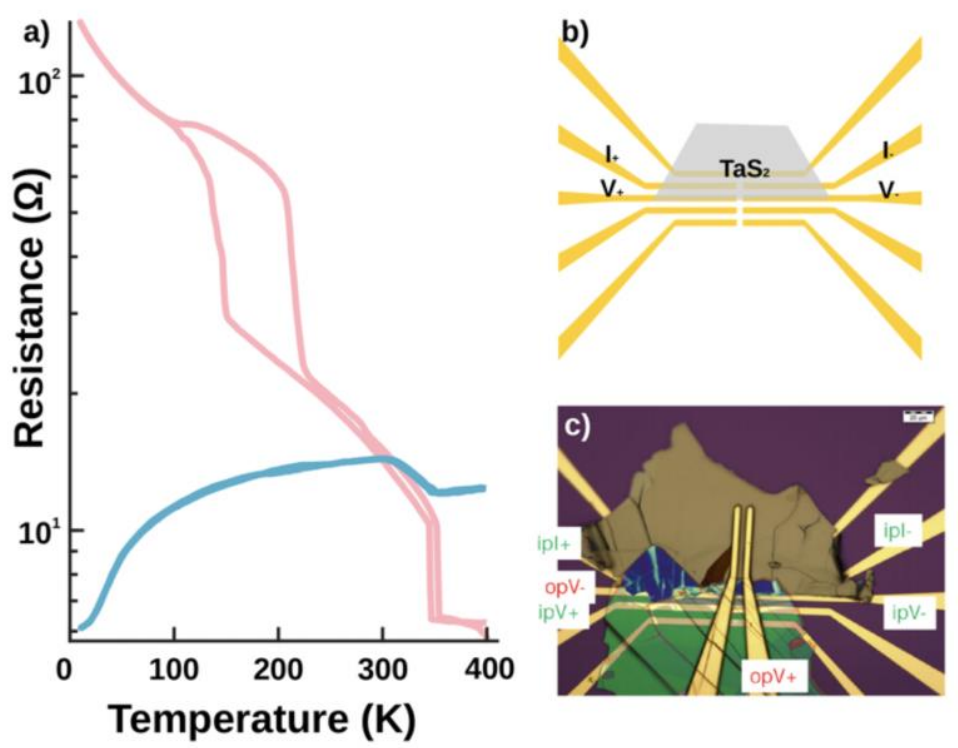

Figure 2. Resistance as a function of temperature for TaS2 devices. a) In-plane resistance measurement of pristine flake (pink) shows expected CDW behavior with two phase transitions. However, disordered stacking and bond coordination (blue) causes dramatically different electronic behavior.

\section{References}

[1] J. A. Wilson, F. J. D. Salvo, and S. Mahajan, Adv. Phys. 24, no. 2, pp. 117-201, (1975)

[2] D. Sakabe, Z. Liu, K. Suenaga, K. Nakatsugawa, S. Tanda, Npj Quantum Mater. 2, p. 22, (2017)

[3] H. Dai and C. M. Lieber, Phys. Rev. Lett., vol. 69, no. 10, pp. 1576-1579, (1992)

[4] T. Ritschel et al., Phys. Rev. B 87, no. 12, p. 125135, (2013)

[5] A. W. Tsen et al., Proc. Natl. Acad. Sci. 112, no. 49, pp. 15054-15059, (2015)

[6] R. Hovden, A. W. Tsen, B. H. Savitzky, P. Liu, I. El Baggari, Y. Liu, W.J. Lu, Y. Sun, P. Kim, A. N. Pasupathy, L. F. Kourkoutis Proc. Natl. Acad. Sci. U.S.A. 113, 11420 (2016)

[7] B. H. Savitsky et al., Nature Communications 8, 1883 (2017)

[8] I. El Baggari et al., Proc. Natl. Acad. Sci. 115, 1445 (2018), p. 1445-1450.

[9] This work was supported by the Keck Foundation 